\title{
Striae Distensae After Breast Augmentation
}

\author{
Nina S. Naidu
}

Received: 19 September 2012/Accepted: 29 November 2012/Published online: 8 January 2013

(C) Springer Science+Business Media New York and International Society of Aesthetic Plastic Surgery 2013

Level of Evidence $V$ This journal requires that authors assign a level of evidence to each article. For a full description of these Evidence-Based Medicine ratings, please refer to the Table of Contents or the online Instructions to Authors http://www.springer.com/00266

I read with great interest the article by Basile et al. [1] "Striae distensae after breast augmentation". Although I agree with their statement that "a clear understanding of the current benefits, risks, and potential complications is essential to providing accurate and complete consultations to patients," I have some questions about the study variables and analysis.

The authors note that the onset of striae distensae (SD) was related to parity, body mass index (BMI), oral contraceptive use, and a history of SD, but they found no association with implant size or type. However, a review of Table 2 shows that all patients received round high-projection or round extra-high-projection implants. Although the range of implant sizes in patients with SD was not excessively large $(235-350 \mathrm{cc})$, the patients' base width and other soft tissue measurements are not given, and therefore the reader is unable to determine if implants too large for the breast tissues might have been used in these cases. Indeed, the safety and efficacy of high- and extrahigh-profile implants remains under debate. Tebbetts and Teitelbaum [2] have argued that the greater size and projection of these implants potentially can have greater negative effects on patients' tissues, including parenchymal thinning, skin stretch, inability to lactate, sensory compromise, implant edge or shell visibility, visible traction rippling, and chest wall deformities. They cite patients at higher risk for these complications, specifically, patients with a tighter skin envelope and patients with previously stretched and thinned tissues. Although deformational changes such as SD potentially can occur with any implant placed into tissues that are tight and cannot accommodate it based on base width and soft tissue stretch of the breast, one wonders if the findings of Basile et al. would have been different had they also included patients with moderateand low-profile implants in their study.

There are a large number of variables that come into play when examining breast augmentation surgery and results, and certainly the authors were able to identify several that were significantly associated with the onset of SD based on their analysis. However, it is not clear if these items would have remained significant had a multivariate analysis been run. Nonetheless, the authors have provided us a valuable service in identifying striae distensae as another potential risk of breast augmentation that should be included routinely on informed consent documents.

Disclosures The author has served as a consultant for Allergan USA, Inc., and is a stock holder in Ideal Implant, Inc.

\section{References}

1. Basile FV, Basile AV, Basile AR (2012) Striae distensae after breast augmentation. Aesthetic Plast Surg 36:894-900

2. Tebbetts JB, Teitelbaum S (2010) High- and extra-high projection breast implants: potential consequences for patients. Plast Reconstr Surg 126:2150-2164 